\title{
Evaluation of 126 Consecutive Stereotactic Procedures: Brain Biopsy, Diagnostic Yield, Accuracy, Non-Diagnostic Results, Complications and Follow-up
}

\author{
Tayfun HAKAN ${ }^{1}$, Fugen VARDAR AKER ${ }^{2}$ \\ ${ }^{1}$ Haydarpasa Numune Teaching and Research Hospital, Department Neurosurgery, Istanbul, Turkey \\ ${ }^{2}$ Haydarpasa Numune Teaching and Research Hospital, Department of Pathology, Istanbul, Turkey
}

\section{ABSTRACT}

AIM: A retrospective analysis of 126 consecutive computed tomography (CT)-guided, frame-based stereotactic procedures in 121 patients is presented to evaluate the diagnostic yield, accuracy, complications, management of non-diagnostic cases and followup.

MATERIAL and METHODS: The medical records of the identified patients were investigated retrospectively. Age, sex, surgical procedures, histopathological diagnosis, diagnostic yield, accuracy, complications, management of non-diagnostic cases and follow-up were analyzed in 121 consecutive patients. Stereotactic procedures were performed by the author by using Leksell's stereotactic system, and stereotactic biopsies were conducted under local anesthesia except for those patients who were not able to tolerate this treatment. These patients had control CT scans two hours after the operation.

RESULTS: Patient age ranged from 2 to 82 years (mean 48 years). Stereotactic biopsy was performed in 112 patients. Cyst and abscess aspiration, intracystic catheter replacement and tumor resection with stereotactic craniotomy were among the other procedures. The diagnostic yield was $93 \%$, and the histological accuracy was $63 \%$ with no mortality. Craniotomy and hematoma evacuation were required in two cases. The patients were followed up from one month to 17 years.

CONCLUSION: Frame-based stereotactic biopsy is a safe and efficacious method with acceptable complications. Experience is important, but not sufficient for preventing complications and performing procedures accurately. Necrosis and gliosis are the most common non-diagnostic findings. Empirical treatment with presumptive diagnoses based on clinical and radiological findings and close clinical follow-up may not affect patients adversely. The follow-up of patients through examination and imaging is important to allow the revision of treatment when necessary.

KEYWORDS: Brain biopsy, Brain tumor, Diagnostic yield, Follow-up, Non-diagnostic biopsy, Stereotactic biopsy

\section{INTRODUCTION}

$\mathrm{E}$ xact histological diagnoses are still important and are strongly recommended when deciding on specific, appropriate and effective treatment options for brain lesions. Most of the treatment protocols for chemo and/or radiotherapy require a histological diagnosis for brain tumors (15). Stereotactic brain biopsy is an effective tool for obtaining tissue samples from unidentified intracranial lesions for adequate therapy (34). Stereotactic surgery started with the Horsley-Clarke apparatus in 1908 (14). After the introduction of stereotactic surgery in human patients by Spiegel and Wycis in 1947, this approach has been used safely and effectively to obtain tissue diagnosis for intracranial lesions. There are many papers establishing the issues related to the safety, accuracy and diagnostic yield of stereotactic biopsy in the literature $(6,9,10,24,28,31,34)$. 
In this paper, a series of stereotactic procedures in 121 consecutive patients is presented and their diagnostic yield, accuracy of stereotactic biopsies, complications, and management and follow-up of non-diagnostic cases are reviewed. To our knowledge, this is the first stereotactic brain biopsy series with a long-term follow-up.

\section{MATERIAL and METHODS}

In this study, 126 consecutive stereotactic procedures that were performed in 121 patients by the author (TH) in Haydarpasa Numune Teaching and Research Hospital were analyzed retrospectively. Informed patient consent was obtained prior to the procedure being carried out from all patients or their legal representatives/guardians. The procedures were carried out using Leksell's stereotactic system (Elektra Instruments, Atlanta, GA, USA) with the computed tomography (CT) images taken by spiral CT scans. Contrast-enhanced CT images were used for the identification of targets and the establishment of stereotactic coordinates. All patients received an antiepileptic medication before the procedure and routinely submitted to a control CT within two hours postoperatively in order to detect any complication with bleeding and to confirm that the biopsy had been taken from its selected target. Local anesthesia was used for the biopsy unless the patient could not tolerate this approach; general anesthesia was used for patients undergoing craniotomy. None of the patients received antiplatelet agents prior to procedure. Other issues related with imaging, targeting, biopsy procedures, specimen preparation and pathological evaluation have been described previously (2).

The cases were separated into biopsy cases and craniotomy cases. Biopsy cases included those involving a stereotactic biopsy, aspiration of cyst or abscess enabling diagnosis, whereas craniotomy cases called for a small craniotomy $(\leq 4$ $\mathrm{cm}$ in diameter) and resection of the lesion under the guidance of a stereotactic needle.

The histological diagnoses based on the two procedures, biopsy \& open resection, were categorized as complete agreement or disagreement to confirm whether they were found to be identical.

\section{RESULTS}

The 121 patients included 76 males and 45 females. The mean age of all of the patients was 48 years, with a range of 2 to 82 years. The lesions were lobar in $61 \%$ and deep-seated in 29\% (Table I).

\section{Stereotactic Procedures}

Stereotactic biopsy was performed in 112 of 126 procedures in 121 patients. One patient had two craniotomies; one patient had biopsy and cyst aspiration with intracystic catheter replacement three times; one patient underwent abscess aspiration two times; and one patient had a biopsy four years after the previous procedure. In 14 cases, craniotomy (one case receive a craniotomy twice) and tumor resection were performed as initial procedures. In one patient, biopsy
+ ventriculoperitoneal (VP) shunt placement were performed. Only in 19 cases were craniotomy and tumor resection followed stereotactic biopsy; the poor clinical condition of the patient, difficulties in the craniotomy and tumor resection and returning to the doctors who referred for biopsy alone were the main causes of this limited number of resections after biopsy.

\section{Diagnostic Yield and Accuracy}

The pathological diagnoses of all biopsies are presented in Table II. The samples were diagnostic in 104 cases and in 112 biopsy cases. The diagnostic yield, which represents the percentage of cases with a definitive histological diagnosis during the biopsy procedure, was $93 \%$. The accuracy of the histological diagnosis was $63 \%$ in 19 cases that underwent open resection in this series.

\section{Misdiagnosed Cases}

The details of misdiagnosis are the business of a paper more focused on pathology; here, clinical reflections were stressed. Still, three cases of major diagnostic error were detected in this series.

In the first case, the complete choroid plexus located in right lateral ventricle was pulled out completely, as if it were a blackberry, with a spiral biopsy needle, and the pilocytic astrocytoma surrounding it was misinterpreted (Figure 1). The pathology of the resection material gave an accurate diagnosis of pilocytic astrocytoma. A side-cutting Sedan aspiration cannula was used in all cases after the biopsy.

In the second case, a 51-year-old patient with a cyst located in the sellar and suprasellar region underwent biopsy, cyst

Table I: Location of the Lesions

\begin{tabular}{cc}
\hline Biopsy cases & No. patients (\%) \\
\hline Lobar & $\mathbf{7 5}(\mathbf{6 7 )}$ \\
\hline Frontal & 38 \\
\hline Parietal & 19 \\
\hline Temporal & 16 \\
\hline Occipital & 3 \\
\hline Deep-seated & $37(33)$ \\
\hline Thalamus & 17 \\
\hline Basal ganglia/corpus callosum & 10 \\
\hline Sellar/suprasellar & 5 \\
\hline Intraventricular & 4 \\
\hline Brain stem & 1 \\
\hline Total & $\mathbf{1 1 2 ( 1 0 0 )}$ \\
\hline Craniotomy cases & $\mathbf{1 4}(\mathbf{1 0 0 )}$ \\
\hline Frontal & $6(43)$ \\
\hline Parietal & $8(57)$ \\
\hline
\end{tabular}


aspiration and catheter replacement; the cyst liquid was clear and colorless, with rare histiocytes. One year later, cyst fenestration was performed; the character of the fluid was same. Aspiration of the cyst continued during the

Table II: Histological Diagnosis of 126 Procedures

\begin{tabular}{|c|c|}
\hline Biopsy cases & n (\%) \\
\hline Neoplastic cases & $90(81)$ \\
\hline Glioblastoma & 30 \\
\hline Anaplastic astrocytoma, grade III & 17 \\
\hline Astrocytoma, grade II & 8 \\
\hline Anaplastic oligodendroglioma, grade III & 1 \\
\hline Oligodendroglioma, grade II & 5 \\
\hline $\begin{array}{l}\text { Anaplastic mixed oligoastrocytoma, } \\
\text { grade III }\end{array}$ & 1 \\
\hline Pilocytic astrocytoma & 1 \\
\hline Central neurocytoma & 2 \\
\hline Choroid plexus papilloma & 1 \\
\hline Craniopharyngioma & 1 \\
\hline Lymphoma & 11 \\
\hline Plasmacytoma & 1 \\
\hline Metastatic carcinoma & 11 \\
\hline Non-Neoplastic cases & $14(12)$ \\
\hline Abscess & 8 \\
\hline Tuberculous granuloma & 1 \\
\hline Hematoma & 1 \\
\hline Echinococcus multilocularis & 1 \\
\hline $\begin{array}{l}\text { Benign cyst content (consisted with } \\
\text { Rathke's cleft csyt) }\end{array}$ & 3 \\
\hline Non-Diagnostic cases & $8(7)$ \\
\hline Total & $112(100)$ \\
\hline
\end{tabular}

\begin{tabular}{ll}
\hline Craniotomy cases & \\
\hline Glioblastoma & 3 \\
\hline Oligodendroglioma, grade II & 1 \\
\hline Meningioma & 1 \\
\hline Metastatic carcinoma & 6 \\
\hline Lymphoma & 1 \\
\hline Tuberculous granuloma & 1 \\
\hline Total & $\mathbf{1 4}^{\star}$
\end{tabular}

*In one case craniotomy was repeated for technical problems. years; however, recraniotomy and tumor resection revealed craniopharyngioma in this patient when the imaging of the cyst changed. Contrast enhancement of the cyst wall was seen only 12 years after the first cyst's aspiration.

The last patient was admitted with a contrast enhancing lesion in her right parietal cortex with a past history of splenectomy due to the autoimmune hemolytic anemia in her childhood. The stereotactic biopsy diagnosis was changed from "Astrocytoma, Grade II" to "granulomatous cerebritis" after the resection of the lesion with craniotomy. Perilesional reactive gliosis was misdiagnosed as astrocytoma. That patient came again four years later with a lesion in the same location. During these years, she had been diagnosed with granulomatous lymphadenitis of the cervical lymph and portal infective reactions in the liver at different medical centers. A new stereotactic biopsy was performed, but no definitive diagnosis was made. The patient refused further investigations in the hematology-immunology clinic and again was lost to follow up.

\section{Non-Diagnostic Cases}

We discussed the first 6 non-diagnostic cases previously (2), but added additional information based on follow-up (Table IV). There were two new non-diagnostic cases. One of them is discussed above. In that case, the patient was initially misdiagnosed as suffering from 'Astrocytoma, Grade II'. The diagnosis was later changed to "granulomatous cerebritis" after a new stereotactic biopsy was performed. The other case was an 11-year-old girl with multiple cerebral cystic lesions in her brain. No diagnosis was possible after stereotactic biopsy; we aspirated straw yellow fluid with no cells. She was diagnosed as acute disseminated encephalomyelitis (ADEM) two months after the procedure and is still alive three years after the treatment (Figure $2 \mathrm{~A}-\mathrm{C}$ ).

\section{Complications}

There was no fatal complication in this study. Overall, we had 8 (6.3\%) complications in 126 procedures; 7 in 112 biopsies and one in 14 craniotomy cases. Small and nonsymptomatic hemorrhages in the biopsy region were detected in $31(27.6 \%)$ of the patients. A generalized seizure attack in one case, progression of hemiparesia in two patients, a minor intraventricular hemorrhage in three cases and a hyperacute intracerebral hemorrhage were the complications in the 112 biopsy cases. Although an early (within two hours) followup CT was normal, an epidural hemorrhage after 17 hours following the craniotomy was the only complication in the craniotomy cases (Figure $3 A-C)$. The seizure, hemiparesis and minor intraventricular hemorrhage cases were treated medically. The other two patients who bled had surgical hematoma evacuations.

\section{Follow-Up}

Clinical follow up after the stereotactic procedures ranged from one month to 17 years. Patients with glial tumors encountered some special difficulties in the follow-up within this study. Most of the patients were referred from other medical centers and were under control of different clinics. Here, the survival 
time was calculated from the time of diagnosis to the time of death (13).

Glial tumors: Glioblastoma was the cause of death in 23 of 33 glioblastoma cases; one patient died due to suicide. The survival time of 23 patients changed between 2-9 months. One patient was still alive after 10 months during the study. Eight glioblastoma patients were lost follow up. Nine out of 17 astrocytoma grade III cases died due to disease, and their

Table III: Comparison of the Diagnosis in 19 Resected Cases

\begin{tabular}{|c|c|c|}
\hline No. of cases (\%) & Biopsy pathology & Resection pathology \\
\hline 1 & Glioblastoma & Glioblastoma \\
\hline 1 & Anaplastic astrocytoma, grade III & Anaplastic astrocytoma, grade III \\
\hline 2 & Astrocytoma, grade II & Astrocytoma, grade II \\
\hline 1 & Oligodendroglioma, grade III & Oligodendroglioma, grade III \\
\hline 2 & Central neurocytoma & Central neurocytoma \\
\hline 1 & Craniopharyngioma & Craniopharyngioma \\
\hline 1 & Metastatic adenocarcinoma & Metastatic adenocarcinoma \\
\hline 1 & Abscess & Abscess \\
\hline 1 & Gemistocytic astrocytoma, grade II & Mixed oligoastrocytoma, grade II \\
\hline 1 & Astrocytoma, grade II & Oligodendroglioma, grade II \\
\hline 1 & Astrocytoma, grade II & Dysembryoplastic neuroepithelial tumour \\
\hline 1 & Astrocytoma, grade II & Granulomatous cerebritis \\
\hline 1 & Choroid plexus papilloma & Pilocytic astrocytoma \\
\hline 1 & Ratke's cleft cyst & Craniopharyngioma (12 years later) \\
\hline
\end{tabular}

Table IV: Non-Diagnostic Cases and Their Management and Follow-up Results

\begin{tabular}{|c|c|c|c|}
\hline $\begin{array}{c}\text { Case } \\
\text { no }\end{array}$ & Stereotactic diagnosis & Management & Follow-up \\
\hline 1 & $\begin{array}{l}\text { Atypical gliosis (low-grade astrocytoma ?/ reactive } \\
\text { gliosis ?) }\end{array}$ & Cons. & $\begin{array}{l}\text { Alive with persistence in the size of lesion } \\
\text { until } 7 \text { years; after that progression of tumor } \\
\text { mass and operation, the new diagnosis was } \\
\text { "anaplastic astrocytoma, grade III" }\end{array}$ \\
\hline 2 & $\begin{array}{l}\text { Reactive gliosis, cerebritis (consisted with peripheric } \\
\text { abscess zone) }\end{array}$ & Mx & $\begin{array}{l}\text { No Evidence of disease after } 11 \text { years and } \\
\text { then follow-up loss }\end{array}$ \\
\hline 3 & $\begin{array}{l}\text { Atypical gliosis and necrosis (consisted with peripheral } \\
\text { tumor zone) }\end{array}$ & $\mathrm{RT} x$ & Died of disease after 6 months \\
\hline 4 & Necrosis (consisted with caseous necrosis) & $\mathrm{Mx}$ & $\begin{array}{l}\text { No evidence of disease after } 32 \text { months and } \\
\text { then follow-up loss }\end{array}$ \\
\hline 5 & Necrosis of tumor (undifferentiated malignant neoplasm) & $\mathrm{RTx}$ & Died of disease after 3 months \\
\hline 6 & Necrosis of tumor (undifferentiated malignant neoplasm) & $\mathrm{RT} x$ & Died of disease after 6 months \\
\hline 7 & Acellular cyst material & Cons. & $\begin{array}{l}\text { Diagnosed as ADEM after one month of } \\
\text { biopsy and alive still for } 3 \text { years }\end{array}$ \\
\hline 8 & No diagnosis (Cerebritis ?) & Cons. & $\begin{array}{l}\text { Refused further investigations and follow-up } \\
\text { loss }\end{array}$ \\
\hline
\end{tabular}

Cons: Conservative, Mx: Medication, RTx: Radiation therapy, ADEM: Acute disseminated encephalomyelitis. 

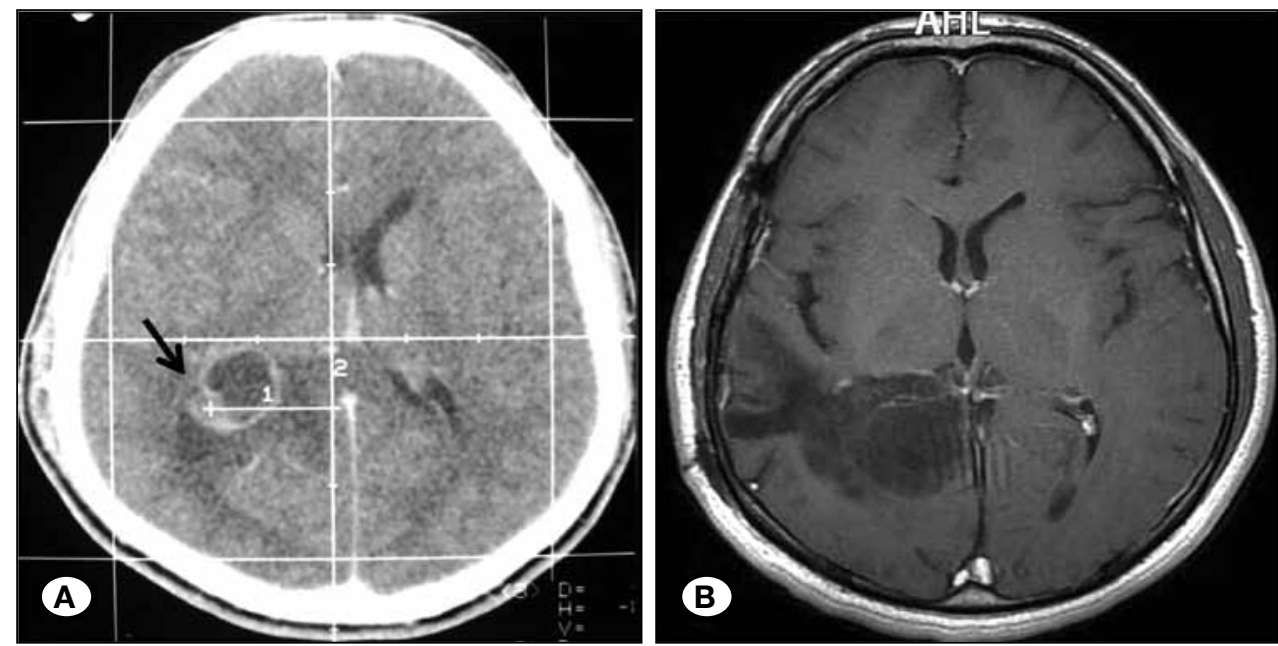

Figure 1: A) A cystic lesion with a mural nodule on CT before stereotactic biopsy (arrow), B) A T1 axial MR image using gadolinium in the same patient after 16 years.
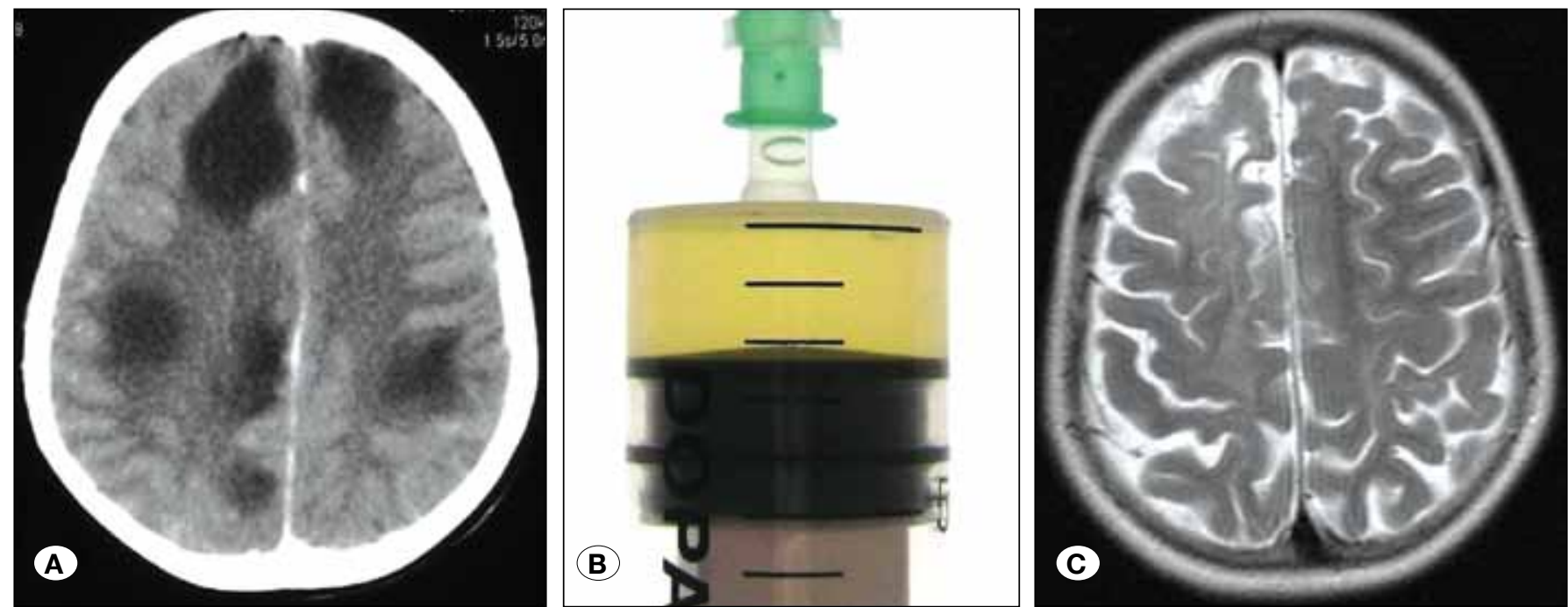

Figure 2: A) Multiple cystic lesions on CT, B) Yellowish and clear acellular fluid aspirated from the right frontal cyst, C) T2 Axial MR imaging using gadolinium in the same patient after 3 years.
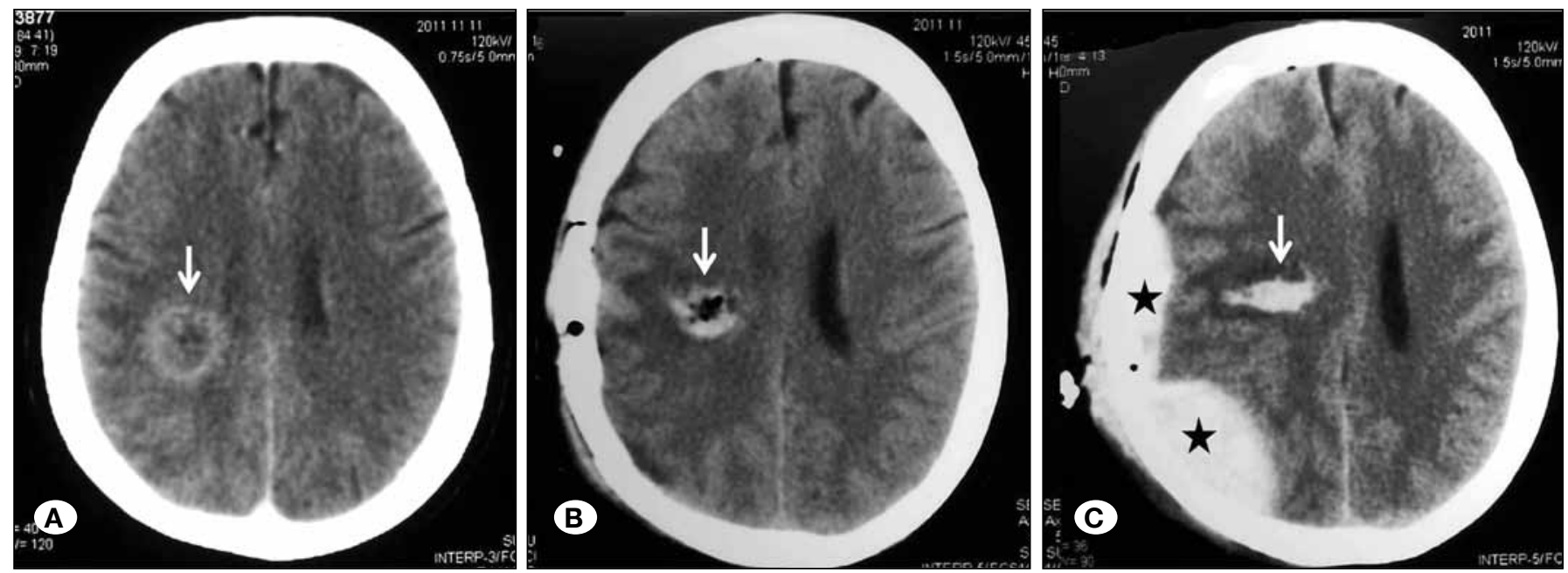

Figure 3: A) A round lesion located in the posterolateral of right lateral ventricle (arrow), B) Minimal postoperative hemorrhage in the tumor cavity on the control CT after 2 hours (arrow), C) Dense hemorrhage in the tumor cavity (arrow) and epidural hematoma (stars) in the control CT after 17 hours. 
survival times were between 1-39 months. Eight patients were lost to follow up. The survival times were 54 and 120 months in two of the 8 astrocytoma grade II patients (Figure 4A-D). One patient with thalamic astrocytoma lived only one month. One patient died immediately, and one patient after 16 months lost follow up. The other three cases were misdiagnosed (Table III). Two oligodendroglioma grade II patients died after 50 and 57 months of biopsy, and the previous patient was the only one with hemorrhaging during biopsy that required an evacuation with craniotomy. Three others were lost to followup. Two mixed oligodendroglioma grade III patients died after 6 and 8 months, respectively. One mixed oligodendroglioma grade II patient was lost to follow-up after 36 months. Both of the pilocytic astrocytoma cases are alive after 16 and 17 years, respectively.

Metastatic carcinomas: Eleven of the 15 patients with a diagnosis of metastatic carcinoma died from the disease within 2-27 months. One patient who had pneumenectomy and oncologic therapy previously was still alive after 56 months after craniotomy and metastatic carcinoma excision. Three of these patients were lost to follow-up.
Lymphomas: Seven of the 11 lymphoma cases died due to the disease. Four lived only 1-2 months, whereas the remaining three patients lived 30, 42 and 68 months, respectively. Four of the lymphoma patients were immediately lost to follow-up, and one patient, after 36 months.

Other tumors: One case of central neurocytoma was still alive after 15 years; unfortunately, the other case died after craniotomy due to the hospital infection. A case of craniopharyngioma was lost to follow-up after 13 years. A case of meningioma was lost to follow-up after 42 months.

Non-neoplastic cases: Only two patients died of other diseases, namely Rathke's cleft cyst and Echinococcus multilocularis. The Rathke's cleft cyst case was diagnosed as craniopharyngioma after 12 years and died one month later due to hypothalamic failure. The other case was published previously as a case report (17).

The survival time was short in high-grade tumors and moderately longer in low-grade tumors, as expected. For nonneoplastic issues, no patient died of disease during the long follow-up period except for two particular cases.
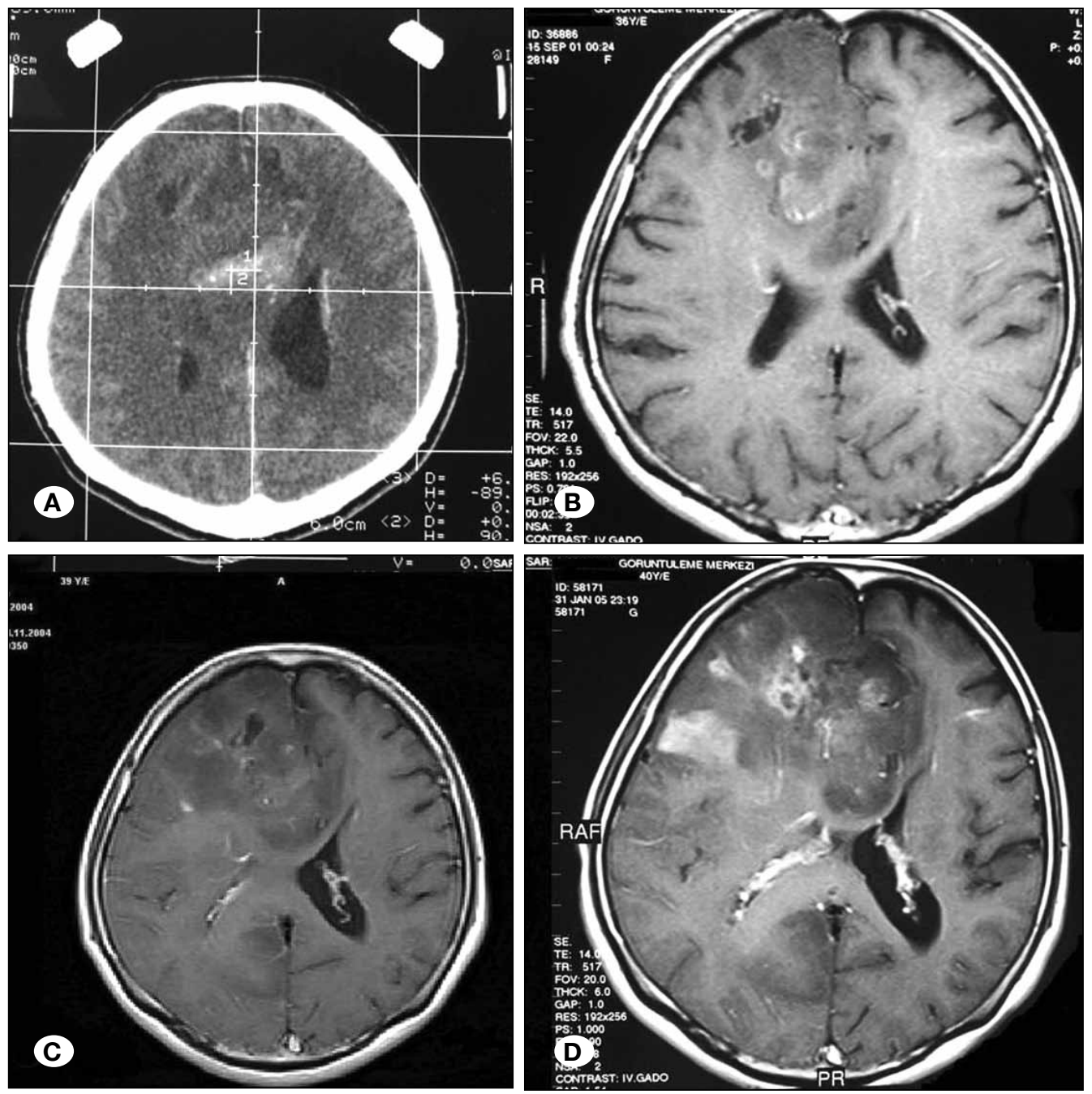

Figure 4: A) Selected target of the lesion for stereotactic biopsy on CT, B) Axial MR imaging using gadolinium in the same patient after 2 years, C) Axial MR imaging using gadolinium in the same patient after 5 years, D) Axial MR imaging using gadolinium in the same patient after 6 years. This patient died 4 years after the final MR investigation but rejected any treatment or radiological investigation during this period. 


\section{DISCUSSION}

All of the experiences were from a single author $(\mathrm{TH})$ with frame-based stereotactic procedures and were retrospectively reviewed. Brain biopsy $(2,17,19)$, aspiration of abscesses $(18)$, accurate placement of intraventricular shunts (19) and/or intracystic catheter, and tumor resection via a small craniotomy with minimal morbidity were among the procedures performed in this study.

\section{Diagnostic Yield and Accuracy}

Many factors affecting the diagnostic yield and accuracy of the stereotactic biopsies have been reported, including the use of a standardized procedure (10), the use of an intraoperative indicator (35), measurements of the lesions (36), the experience of neurosurgeons and neuropathologists $(21,36)$, intraoperative smear examinations (37), tissue heterogeneity (38), outside review of the pathological slides and further tissue samplings (39). Tilgner et al. (34) compared a huge population of intraoperative and final diagnosis and found that the accuracy was above average in the lesions located in the occipital lobe and lesions with multiple locations. Shooman et al. (32) obtained a high diagnostic yield of 99.3 percent despite their omission of confirmatory intraoperative neuropathological assessments. Jain et al. (22) suggested that multiple bits were associated with a high diagnostic yield, even reaching $100 \%$ for 5 to 6 bits; however, Zoeller et al. (39) found no statistically significant correlation between the number of biopsies and non-diagnosis. The diagnosis and grading of glial tumors are special applications of the stereotactic biopsy $(2,5,13,21,23,27)$, and one of the main problems is the representation of the whole lesion within the biopsy material $(21,24)$. Callovini $(5)$ concluded that, in the lesions of the corpus callosum, histological analysis seems not to be required in order to proceed to complementary therapies, presuming these lesions are high grade gliomas. However, one of our patients with a huge lesion of corpus callosum had a diagnosis of astrocytoma, grade II and refused tumor resection; only submitting to radiotherapy, he lived 10 years before dying of the disease. Grossman et al. (16) speculated that intra-operative bleeding through the biopsy needle may cause a lower diagnostic yield of biopsies, probably as a result of prematurely aborted procedures. It is a fact that intraoperative bleeding through the biopsy needle seriously increases the surgeon's anxiety and reluctance to proceed with any further biopsy. Ranjan et al. (29) found no significant difference between the most experienced and other surgeons regarding negative biopsies. Two non-diagnostic cases were the $94^{\text {th }}$ and $125^{\text {th }}$ procedures in the present study.

A stereotactic biopsy diagnosis can result in a difference in $3-49 \%$ of cases after tumor removal (27). It has been shown that intraoperative diagnosis via neurocytology and/or frozen section increases this accuracy from 88 to $95 \%$ (26). Reithmeier et al. (30) achieved a complete correlation of the histopathological findings between the biopsy group and the resection group in $76 \%$ of 51 patients who had stereotactic biopsies and subsequent open resection within 30 days the cases, reporting an increase of $90 \%$ after analyzing the clinical and neuroradiological information. Chernov et al. (7) reported a $100 \%$ diagnostic yield in spectroscopy-supported framebased stereotactic biopsies of the parenchymal brain lesions; this yield was found to be $90 \%$ in MRI-supported procedures. The overall diagnostic accuracy was $73 \%, 67 \%$ and $79 \%$ for spectroscopy- and MRI-supported biopsies, respectively, in their series. They found a diagnostic accuracy of $35 \%$ in 37 patients, with the minor and major disagreements of $38 \%$ and $27 \%$, respectively. Other series have reported the complete correlation of the first and final diagnosis between $52 \%$ and $81.3 \%(8,12,22)$.

Woodworth et al. (38) reported that, although the accuracy was $76 \%$ in their series, the biopsy results correctly guided the treatment in $91 \%$ of the patients. Similarly, despite the complete agreement found in $63 \%$ of the presented series, only one patient's treatment was affected, altering his biopsy diagnoses from Rathke's cleft cyst to craniopharyngioma after 12 years. The expected survival time may provide some clue about the accuracy or correctness of the final diagnoses. The survival times of the cases were found to be in accordance with the final diagnoses in this study.

\section{Non-Diagnosis and Misdiagnosis}

When a definitive histological or microbiological diagnosis cannot be obtained, it is accepted as a non-diagnostic biopsy, with rates reported between 3-19 \% (1,29,33,39). Many predictive factors for non-diagnostic biopsy have been described, including younger age $(11,23)$, longer operating time (10), insufficient material and sampling error (4), lesions locating at right side (10), midline and cerebellum $(9,25)$, smaller and deeply located lesions and posterior fossa lesions (3). A misdiagnosis is an incorrect diagnosis and occurs most often with mis-staged glial tumors during stereotactic brain biopsies (36). In the present series, no predictive factors were detected for non-diagnostic lesions. Tilgner et al. (34) speculated that such complex entities as neurocytomas can cause misdiagnosis; we encountered two neurocytoma diagnoses that had their accuracy confirmed by open resection in this series. Waters et al. (36) suggested that risk of a nondiagnostic biopsy for $<1 \mathrm{~cm}^{3}$ lesions is significantly higher than for general lesions. On the other hand, Heper et al. (20) indicated that the amount of tissue sampled did not decrease the diagnostic yield using new stereotactic technologies. Necrosis and the improper quality of smear preparations can cause difficulties establishing a histopathologic diagnosis in stereotactic biopsies. Ranjan et al. (29) classified nondiagnostic biopsies as negative and inconclusive; the former included those that failed to indicate the nature of the mass, and the latter, those that showed a tissue but were unable to facilitate a definitive diagnosis. The authors reported that gliosis and necrosis were the two most common pathological findings among the negative biopsies. Gliosis and necrosis were also common in the present series. Out of 3 cases of necrosis, two of were presumed to be undifferentiated tumors, one of which was consistent with caseous necrosis. In cases of non-diagnostic diagnosis, the continuation of the procedure could cause not only futile biopsies but also, more importantly, can lead to potentially catastrophic consequences (32). 


\section{Management and Follow-Up of Non-Diagnostic Cases}

There are few studies on the management and follow up of non-diagnostic biopsy cases in the literature $(1,13,23,29,39)$. The institution of empirical treatments after a presumptive diagnosis using serial imaging was advised for patients with non-diagnostic biopsies $(1,39)$. In this series, two patients were treated with antibiotics and antituberculosis agents successfully; they lived 11 years and 32 months, respectively, before their loss to follow up. Patients with an inconclusive diagnosis of neoplasm consistent with a peripheral tumor zone or of necrosis of the tumor consistent with an undifferentiated malign neoplasm received radiotherapy, as advised previously (29).

Gaudin et al. (13) followed patients with diagnosis of glioma for over 2 years and concluded that the diagnosis and grading of gliomas with stereotactic biopsies provided reliable prognostic information. From another point of view, following up patients who submit to stereotactic biopsies may further prove the correctness of the diagnosis. The follow-up and survival time of the patients in this study indicated such a result based on their collection of correct and wrong diagnoses. After clinical follow-up, Zoeller et al. (39) found that non-diagnostic biopsies did not affect their patients adversely. In this study, patients with non-neoplastic and low-grade lesions we observed to have a prolonged survival with a long follow-up. The patient diagnosed with necrosis (consisting of caseous necrosis) lived 32 months until loss to follow-up. One patient with a diagnosis of atypical gliosis and low grade astrocytoma progressed to a diagnosis of anaplastic astrocytoma. One of two cerebritis cases lived 11 years free of disease until loss to follow-up. The other "cerebritis" case was a previous patient of ours who had this diagnosis and lived for 4 years. The last patient with nondiagnosis was diagnosed with ADEM after further research. Khatab et al. (23) found relatively lower survival rates for glioblastoma patients, similar to the low rates in the presented series. The patient who had a diagnosis of atypical gliosis and necrosis with a peripheral tumor zone died of disease after 6 months, and the two patients who had tumor necrosis (undifferentiated malignant neoplasm?) died of disease after 3 and 6 months, respectively. The patients with brain biopsies should be considered different from the general population of brain tumors. The clinical situation, characteristics of the tumor (ineligibility of tumor, multiplicity, location, etc.), patient preferences, and management of the patients by different medical centers and/or physicians may influence their survival. Overall, all of the patients died or lived in a manner compatible with the presumed diagnosis and management in this study.

\section{Hemorrhagic Complications}

One of the most important complications of stereotactic brain biopsies is hemorrhage; whether symptomatic or not, its importance lies in its potential fatality in some conditions (16). To minimize the risk of hemorrhage, no further biopsy samples should be taken after the pathologist makes a diagnosis (34). Any opportunity for intraoperative diagnosis is also an advantage to avoid this type of complications. We stopped the biopsies as soon as the pathologist was able to give an intraoperative diagnosis, especially in patients who bled through the biopsy needle in this series; this approach may have prevented us from any fatal hemorrhagic complications. Still, it is obvious that non-diagnoses or misdiagnoses are always better than death with an accurate diagnosis.

Lesions located at the brain stem $(11,16)$, pineal region, sylvian fissure, periventricular regions and subarachnoid space are considered to carry a higher risk (11). Reithmeier et al. (30) reported a $2 \%$ mortality as a result of a fatal bleeding and an $8 \%$ silent bleeding rate without any clinical symptoms in a recent series. Smith et al. (33) reported that there were no significant differences regarding the incidence of complications between frame-based and frameless biopsy. Platelet counts below 150,000/ $\mathrm{mm}^{3}$ (11), liver cirrhosis (6), deep location and lymphoma (25) were found to be increased risk factors for hemorrhage. Owen et al. (28) reported that all cases bleeding through the biopsy needle were able to be stopped by continuing irrigation. In the presented study, the patient who bled during biopsy developed an arterial hemorrhage that could not be stopped, and the patient immediately lost consciousness with signs of uncal herniation before undergoing a hematoma evacuation. Field et al. (11) reported a post-biopsy intra-parenchymal hemorrhage, even after 24 hours. In the series of Grossman et al. (16), the occurrence of delayed deterioration due to parenchymal hematoma was detected not only after several hours but also within several days. In the present series, the patient who underwent a small craniotomy and tumor resection developed an epidural hematoma in the craniotomy field 17 hours after the operation, even though there were no signs of bleeding on the postoperative CT after two hours. Chen et al. (6) found a higher incidence of complications after burr-hole craniotomy, but the complications were not related with hemorrhage by nature. Two severe complications involving bleeding that required craniotomy were the $104^{\text {th }}$ and $122^{\text {nd }}$ cases in the present study. Physician experience was not able to avoid any of the major complications, non-diagnoses or misdiagnoses; it is clear that every new case should be managed individually and meticulously. In one case, the spiral biopsy needle pulled out the whole choroid plexus located in lateral ventricle. No major complication arose other than a small hemorrhage not requiring a surgical intervention, and we used a side-cutting Sedan biopsy cannula after this event.

\section{- CONCLUSION}

In the present retrospective series, frame-based stereotactic biopsy was shown to be a safe and efficacious method with acceptable complications. Experience is important but not sufficient for preventing complications and achieving accuracy. Necrosis and gliosis are the most common findings in nondiagnostic cases. Empirical treatments with presumptive diagnoses based on clinical and radiological findings and close clinical follow-up may not affect patients adversely. The follow-up of patients through examination and imaging is important to allow the revision of treatment when necessary. 


\section{- REFERENCES}

1. Air EL, Warnick RE, McPherson CM: Management strategies after nondiagnostic results with frameless stereotactic needle biopsy: Retrospective review of 28 patients. Surg Neurol Int 3:S315-319, 2012

2. Aker FV, Hakan T, Karadereler S, Erkan M: Accuracy and diagnostic yield of stereotactic biopsy in the diagnosis of brain masses: Comparison of results of biopsy and resected surgical specimens. Neuropathology 25:207-213, 2005

3. Barnett GH, Miller DW, Weisenberger J: Frameless stereotaxy with scalp-applied fiducial markers for brain biopsy procedures: Experience in 218 cases. J Neurosurg 91:569576, 1999

4. Calişaneller T, Ozdemir O, Ozger O, Ozen O, Kiyici H, Caner H, Altinörs N: The accuracy and diagnostic yield of computerized tomography guided stereotactic biopsy in brain lesions. Turk Neurosurg 18:17-22, 2008

5. Callovini GM: Is it appropriate to redefine the indication for stereotactic brain biopsy in the MRI Era? Correlation with final histological diagnosis in supratentorial gliomas. Minim Invasive Neurosurg 51:109-113, 2008

6. Chen CC, Hsu PW, Erich Wu TW, Lee ST, Chang CN, Wei KC, Chuang CC, Wu CT, Lui TN, Hsu YH, Lin TK, Lee SC, Huang YC: Stereotactic brain biopsy: Single center retrospective analysis of complications. Clin Neurol Neurosurg 111:835839, 2009

7. Chernov MF, Muragaki Y, Ochiai T, Taira T, Ono Y, Usukura M, Maruyama T, Nakaya K, Nakamura R, Iseki H, Kubo O, Hori T, Takakura K: Spectroscopy-supported frame-based imageguided stereotactic biopsy of parenchymal brain lesions: comparative evaluation of diagnostic yield and diagnostic accuracy. Clin Neurol Neurosurg 111:527-535, 2009

8. Collaço LM, Tani E, Lindblom I, Skoog L: Stereotactic biopsy and cytological diagnosis of solid and cystic intracranial lesions. Cytopathology 14:131-135, 2003

9. Dammers R, Haitsma IK, Schouten JW, Kros JM, Avezaat CJ, Vincent AJ: Safety and efficacy of frameless and frame-based intracranial biopsy techniques. Acta Neurochir (Wien) 150:2329, 2008

10. Dammers R, Schouten JW, Haitsma IK, Vincent AJ, Kros JM Dirven CM: Towards improving the safety and diagnostic yield of stereotactic biopsy in a single centre. Acta Neurochir (Wien) 152:1915-1921, 2010

11. Field M, Witham TF, Flickinger JC, Kondziolka D, Lunsford LD: Comprehensive assessment of hemorrhage risks and outcomes after stereotactic brain biopsy. J Neurosurg 94:545551,2001

12. Firlik KS, Martinez AJ, Lunsford LD: Use of cytological preparations for the intraoperative diagnosis of stereotactically obtained brain biopsies: A 19-year experience and survey of neuropathologists. J Neurosurg 91:454-458, 1999

13. Gaudin PB, Sherman ME, Brat DJ, Zahurak M, Erozan YS: Accuracy of grading gliomas on CT-guided stereotactic biopsies: A survival analysis. Diagn Cytopathol 17:461-466, 1997

14. Gildenberg PL: The birth of human stereotactic surgery. Acta Neurochir Suppl 117: 1-4, 2013
15. Grant R: Overview: Brain tumour diagnosis and management/ Royal College of Physicians guidelines. J Neurol Neurosurg Psychiatry 75 Suppl 2: ii18-ii23, 2004

16. Grossman R, SadetzkiS, Spiegelmann R, RamZ: Haemorrhagic complications and the incidence of asymptomatic bleeding associated with stereotactic brain biopsies. Acta Neurochir (Wien) 147:627-631, 2005

17. Hakan T, Aker FV: A case report of fatal echinococcosis. Annals of Neurosurgery 1(1): 14-19, 2001. http://annalsneurosurgery.org/papers/hakan_t/hakan_t.pdf (accessed in November $10^{\text {th }}$ 2014)

18. Hakan T, Ceran N, Erdem I, Berkman MZ, Göktaş P: Bacterial brain abscesses: An evaluation of 96 cases. J Infect 52: 359366, 2006

19. Hakan T, Berkman MZ, Aker F V: Glioblastoma located in the third ventricle: Case report. J Neurol Sci (Turk) 22:85-88, 2005

20. Heper AO, Erden E, Savas A, Ceyhan K, Erden I, Akyar S, Kanpolat Y: An analysis of stereotactic biopsy of brain tumors and nonneoplastic lesions: A prospective clinicopathologic study. Surg Neurol 64 Suppl 2:S82-88, 2005

21. Jackson RJ, Fuller GN, Abi-Said D, Lang FF, Gokaslan ZL, Shi WM, Wildrick DM, Sawaya R: Limitations of stereotactic biopsy in the initial management of gliomas. Neuro Oncol 3:193-200, 2001

22. Jain D, Sharma MC, Sarkar C, Deb P, Gupta D, Mahapatra AK: Correlation of diagnostic yield of stereotactic brain biopsy with number of biopsy bits and site of the lesion. Brain Tumor Pathol 23:71-75, 2006

23. Khatab S, Spliet W, Woerdeman PA: Frameless image-guided stereotactic brain biopsies: Emphasis on diagnostic yield. Acta Neurochir (Wien) 156(8):1441-1450, 2014

24. Kim JE, Kim DG, Paek SH, Jung HW: Stereotactic biopsy for intracranial lesions: Reliability and its impact on the planning of treatment. Acta Neurochir (Wien) 145:547-555, 2003

25. Livermore LJ, Ma R, Bojanic S, Pereira EA: Yield and complications of frame-based and frameless stereotactic brain biopsy - The value of intra-operative histological analysis. Br J Neurosurg 28(5):637-644, 2014

26. Martinez AJ, Pollack I, Hall WA, Lunsford LD: Touch preparations in the rapid intraoperative diagnosis of central nervous system lesions. A comparison with frozen sections and paraffin-embedded sections. Mod Pathol 1:378-384, 1988

27. Muragaki Y, Chernov M, Maruyama T, Ochiai T, Taira T, Kubo $\mathrm{O}$, Nakamura R, Iseki H, Hori T, Takakura K: Low-grade glioma on stereotactic biopsy: How often is the diagnosis accurate? Minim Invasive Neurosurg 51:275-279, 2008

28. Owen CM, Linskey ME: Frame-based stereotaxy in a frameless era: Current capabilities, relative role, and the positive- and negative predictive values of blood through the needle. $J$ Neurooncol 93:139-149, 2009

29. Ranjan A, Rajshekhar V, Joseph T, Chandy MJ, Chandi SM: Nondiagnostic CT-guided stereotactic biopsies in a series of 407 cases: Influence of CT morphology and operator experience. J Neurosurg 79:839-844, 1993 
30. Reithmeier T, Lopez WO, Doostkam S, Machein MR, Pinsker MO, Trippel M, Nikkhah G: Intraindividual comparison of histopathological diagnosis obtained by stereotactic serial biopsy to open surgical resection specimen in patients with intracranial tumours. Clin Neurol Neurosurg 115:1955-1960, 2013

31. Shakal AA, Mokbel EA: Hemorrhage after stereotactic biopsy from intra-axial brain lesions: Incidence and avoidance. J Neurol Surg A Cent Eur Neurosurg 75(3):177-182, 2014

32. Shooman D, Belli A, Grundy PL: Image-guided frameless stereotactic biopsy without intraoperative neuropathological examination. J Neurosurg 113:170-178, 2010

33. Smith JS, Quiñones-Hinojosa A, Barbaro NM, McDermott MW: Frame-based stereotactic biopsy remains an important diagnostic tool with distinct advantages over frameless stereotactic biopsy. J Neurooncol 73:173-179, 2005

34. Tilgner J, Herr M, Ostertag C, Volk B: Validation of intraoperative diagnoses using smear preparations from stereotactic brain biopsies: Intraoperative versus final diagnosis--influence of clinical factors. Neurosurgery 56:257-265, 2005
35. von Campe G, Moschopulos M, Hefti M: 5-Aminolevulinic acid-induced protoporphyrin IX fluorescence as immediate intraoperative indicator to improve the safety of malignant or high-grade brain tumor diagnosis in frameless stereotactic biopsies. Acta Neurochir (Wien) 154:585-588, 2012

36. Waters JD, Gonda DD, Reddy H, Kasper EM, Warnke PC, Chen CC: Diagnostic yield of stereotactic needle-biopsies of sub-cubic centimeter intracranial lesions. Surg Neurol Int 4:176-181, 2013

37. Winkler D, Lindner D, Richter A, Meixensberger J, Schober J: The value of intraoperative smear examination of stereotaxic brain specimens. Minim Invasive Neurosurg 49:353-356, 2006

38. Woodworth G, McGirt MJ, Samdani A, Garonzik I, Olivi A, Weingart JD: Accuracy of frameless and frame-based imageguided stereotactic brain biopsy in the diagnosis of glioma: Comparison of biopsy and open resection specimen. Neurol Res 27:358-362, 2005

39. Zoeller GK, Benveniste RJ, Landy H, Morcos JJ, Jagid J: Outcomes and management strategies after nondiagnostic stereotactic biopsies of brain lesions. Stereotact Funct Neurosurg 87:174-181, 2009 